\title{
Investigating lymphadenopathy-report on the first 12 months of the lymph node diagnostic clinic at the Royal Marsden Hospital
}

\author{
R K Gregory, D Cunningham, T A Fisher, P Rhys-Evans, G W Middleton, L Bishop, \\ A Wotherspoon, P A Trott, A G Nash
}

\begin{abstract}
The lymph node diagnostic clinic was set up at the Royal Marsden Hospital to provide a direct access service for general practitioners. In the first year 82 patients were seen. The malignancy pick-up rate was $19.5 \%$ which compares very favourably to rates in breast and colorectal clinics. Patient and general practitioner satisfaction with the service was high. (Postgrad Med f 2000;76:566-568)
\end{abstract}

Keywords: rapid diagnosis; lymphadenopathy

Lymphadenopathy is a common problem in general practice and one which can create much anxiety in both patients and their doctors. In the majority of cases the cause of the lymphadenopathy is benign but it can be a presenting sign of a number of serious conditions including haematological malignancies, metastatic carcinoma, connective tissue disorders, and infections such as tuberculosis. At the present time the referral pattern for these patients is varied, most commonly to the local general surgeon for biopsy as a preliminary investigation. It was our impression that this could lead to delays in definitive diagnosis in these patients, which at best is associated with increased anxiety to the patient and at worst could lead to significant delays in starting treatment in patients with serious conditions particularly with malignant disease in the head and neck with potential adverse effects on morbidity and mortality.

In view of this we set up a lymph node diagnostic clinic under the auspices of the Lymphoma Unit at the Royal Marsden Hospital. This is a report on the first year's activity of this clinic.

\section{Methods}

The lymph node diagnostic clinic was set up to run within the existing clinics of the unit which meant that we were able to see patients referred to the service on four days a week. Letters were sent to local general practitioners (GPs) informing them of the service, referrals were received by letter, fax, or phone call, and patients were given an appointment in the next available clinic. All patients were initially seen by members of the Lymphoma Unit when an initial assessment was made, routine blood tests performed, and further investigations organised. On this occasion the majority of patients also had fine needle aspiration of the enlarged node performed. If formal biopsy was felt to be indicated the patients were referred to either the general or ear, nose, and throat (ENT) surgeons within the hospital depending on the site of the node; all patients with suspicious cervical lymphadenopathy were referred for formal ENT examination within one week. All patients were also seen by the Lymphoma Unit research nurse who provided a contact point at the hospital.

We collected data from the Lymphoma Unit database on the patients seen in the first year of the clinic's operation including investigations performed and final diagnosis. Questionnaires were sent out to all patients referred to the clinic inviting comments on patient satisfaction and time to appointment. In addition all doctors referring to the clinic were contacted for their comments on the service.

\section{Results}

In total 82 patients were seen during the first year of the clinic. Of these 43 patients were women. The age of patients referred ranged from 15 to 86 years (median 66). Table 1 indicates the nodal site of concern at presentation. As shown, the majority of patients had cervical node enlargement, with approximately equal numbers of patients with groin, axillary, or multiple nodal sites enlarged. All patients had "lymphoma bloods" taken for full blood count, erythrocyte sedimentation rate, urea and electrolytes, liver function tests, lactate dehydrogenase, and immunoglobulins including B2 microglobulin. Just over half of the patients (42/82) had fine needle aspiration for cytology performed and 21 patients had an excision biopsy. We analysed the cytology results for patients with an eventual malignant diagnosis. Of the 16 patients in this category one proceeded straight to excision biopsy and of the 15 others, 10 (75\%) had a cytology suspicious of malignant diagnosis.

Table 2 details the final diagnoses of patients referred to the clinic, $16 / 82(19.5 \%)$ patients had a malignant diagnosis and the specific malignant diagnoses are also shown in table 2. All patients were brought back to the clinic to receive test results and to discuss their

Table 1 Site of lymph node enlargement in patients referred to the lymph node diagnostic clinic

\begin{tabular}{ll}
\hline Site of nodal enlargement & No of patients \\
\hline Cervical & 53 \\
Axillary & 7 \\
Inguinal & 12 \\
Muliple sites & 10 \\
Total patients & 82 \\
\hline
\end{tabular}

Submitted 5 November 1999 Accepted 10 February 2000 
Table 2 Final diagnoses for patients referred to the lymph node diagnostic clinic

\begin{tabular}{ll}
\hline Diagnosis & No of patients \\
\hline Reactive lymphadenopathy & 51 \\
Malignancy & 16 \\
Lymphoma & 9 \\
Head and neck cancer & 3 \\
Chronic lymphocytic leukaemia & 1 \\
Oesophageal carcinoma & 1 \\
Malignant melanoma & 1 \\
Thyroid carcinoma & 1 \\
Infection & 4 \\
Benign adenoma & 3 \\
Tuberculosis & 3 \\
Infectious mononucleosis & 2 \\
Discoid lupus & 1 \\
Dermopathic lymphadenopathy & 1 \\
Lipoma & 1 \\
\hline
\end{tabular}

diagnosis and further treatment if appropriate; GPs were informed of all results in clinic letters. The Royal Marsden Hospital is a specialist cancer hospital. Patients with a nonmalignant diagnosis were referred directly on to the appropriate specialist service in their local hospital.

\section{QUESTIONNAIRE}

Of the patients contacted $51.6 \%$ returned questionnaires. Unfortunately we have no information on whether or not response was higher in patients receiving a definitive diagnosis as the questionnaires were returned anonymously. Of those returned, $87 \%$ were positive about the clinic and the treatment they received. Of the patients who were less happy several commented on the worry that being referred to a specialist cancer hospital had entailed and one patient was unhappy that she did not undergo computed tomography. In the questionnaire we asked patients for the time they had waited for an appointment: $54 \%$ were seen in the hospital within seven days of visiting their GP and $83 \%$ within 14 days. In many cases patients requested a later appointment due to prior commitments. Four patients were seen outside this time: one patient stated a wait of 28 days, and three a wait of 21 days. We looked into these four cases as we felt that this was an unacceptably long delay and in each of these cases patients were seen within three days of the referral letter being received at the hospital.

Of the GPs referring to the clinic, $76 \%$ responded to the questionnaire and of these $88 \%$ were satisfied and would use the service again, $9 \%$ had no information on the patients as the patient had changed lists, and 3\% said they would use the service again but would have liked more information behind the rationale of tests ordered and guidance on future referrals.

\section{Discussion}

The lymph node diagnostic clinic was set up with the intention of providing a rapid diagnostic service to local GPs. The pick up rate for malignancies was $\sim 20 \%$, but other diagnoses included tuberculosis and discoid lupus. We could find no other reports of the activity of a lymph node diagnostic clinic; however our "malignancy pick-up rate" compares favourably with those seen in breast and colorectal clinics. A report on 2332 patients referred by their GPs to the Rapid Access Breast Diagnostic Clinic at the University Hospital of Wales revealed a $6.3 \%$ malignancy rate. ${ }^{1}$ The St George's rapid access proctology clinic reports a malignancy rate of $2.4 \%{ }^{2}$

The level of satisfaction with the clinic was high, both among the patients referred to the service and among referring doctors. Several patients expressed reservations about being referred to a specialist cancer hospital, as this seemed to provoke anxiety, but others felt that being seen at a specialist centre was reassuring. We are addressing this issue by compiling a patient information leaflet that will be sent out with the patient's appointments and also to referring GPs explaining that the majority of patients referred do not have cancer and detailing what is likely to happen on the clinic visit and subsequent investigations.

Of note, the majority of patients seen had cervical node enlargement. This may in part reflect the fact that patients with nodal enlargement at other sites are referred to other specialists - for example, women with axillary nodal enlargement would most commonly be referred to a breast clinic. Cervical node enlargement can be due to a number of "benign" causes, most typically infection but otherwise asymptomatic head and neck carcinomas are an important alternative diagnosis. Patients with cervical lymph node enlargement persisting over one month should be referred to a head and neck specialist for a formal examination of the aerodigestive tract. Suspicion should be particularly high in patients who are heavy smokers or drinkers. All patients with cervical lymphadenopathy referred to this clinic had an appropriate ENT examination within one week. The site of node enlargement can be suggestive of a primary site in head and neck malignancies. Upper cervical lymphadenopathy, particularly the jugulodigastric node, was more likely to be associated with squamous carcinoma of the oral cavity and pharynx but in this study supraclavicular fossa lymphadenopathy was associated with cases of thyroid and oesophageal carcinoma.

Fine needle aspiration was performed routinely in clinic. Seventy five per cent of patients with an eventual malignant diagnosis had a suspicious or diagnostic fine needle aspiration. Fine needle aspiration is increasingly used as a diagnostic tool and is well established in the diagnosis of carcinoma of the breast and other forms of metastatic carcinoma. However its role in the diagnosis of lymphoma is less certain, and biopsy remains the investigation of choice, although several authors comment on its role in indicating a serious diagnosis and hence expediation of other staging investigations. $^{34}$ All studies report on the importance of operator experience in obtaining adequate fine needle aspirates.

In summary, patient and GP satisfaction with the clinic was high and $\sim 20 \%$ of patients seen had a malignant diagnosis and proceeded to treatment within the appropriate unit at the Royal Marsden Hospital. The clinic continues 
to be run with a referral rate of approximately two patients per week.

1 Cochrane RA, Singhal H, Monneypenny IJ, et al. Evaluation of general practitioner referrals to a specialist breast clinic according to the UK national guidelines. Eur $\mathcal{F}$ Surg Oncol 1997;23:198-2.
2 Boghossian P, Miles WFA, Gudgeon AM, et al. The rapid access proctology clinic: an appraisal of one year's experience. Br f Gen Pract 1996;46:741-2.

3 Suhrland MJ, Wieczorek R. Fine needle aspiration biopsy in the diagnosis of lymphoma. Cancer Invest 1991;9:61-8.

4 Dmitrovsky E, Martin SE, Krudy AG, et al. Lymph node aspiration in the management of Hodgkin's disease. 7 Clin Oncol 1986;4:306-10.

\section{Medical Anniversary}

\section{Richard Bright, 28 September 1789}

Richard Bright (1789-1858) was born in Bristol, son of a successful merchant and banker of substance. He was educated at Edinburgh and Guy's Hospital. He qualified in 1816 and was soon appointed assistant physician at Guy's (1820), where he made his great clinicopathological study of nephritis. One of his diagnostic procedures was to hold a spoonful of urine over a candle and note the development of coagulum; a simple test for albuminuria. His memorable work Reports of Medical Cases, selected with a view of illustrating the Symptoms and Cure of Disease by a reference to Morbid Anatomy was published in 1827 by Longmans and dedicated to his colleagues and friends Benjamin Harrison and William Babington. A trio of brilliant Guy's men-Bright, Addison, and Hodgkin-laid the foundation of modern clinicopathological medicine. Bright died on 16 December 1858 at his home 11 Savile Row, London from an intestinal haemorrhage. He was buried at Kensal Rise and later a mural was placed in his memory in St James' Church, Piccadilly. - D G fames 